\title{
OPEN OCT angiography metrics predict intradialytic hypotension episodes in chronic hemodialysis patients: a pilot, prospective study
}

\author{
Giuseppe Coppolino ${ }^{1,3}$, Adriano Carnevali ${ }^{2,3 凶}$, Valentina Gatti ${ }^{2}$, Caterina Battaglia ${ }^{2}$, \\ Giorgio Randazzo ${ }^{2}$, Irma Figlia ${ }^{1}$, Gemma Patella ${ }^{1}$, Giorgio Fuiano ${ }^{1}$, Michele Andreucci ${ }^{1}$, \\ Giuseppe Giannaccare ${ }^{2}$, Vincenzo Scorcia ${ }^{2}$ \& Davide Bolignano ${ }^{1}$
}

In chronic hemodialysis (HD) patients, intradialytic hypotension (IDH) is a complication that increases mortality risk. We run a pilot study to analyzing possible relationships between optical coherence tomography angiography (OCT-A) metrics and IDH with the aim of evaluating if OCT-A could represent a useful tool to stratify the hypotensive risk in dialysis patients. A total of 35 eyes ( 35 patients) were analyzed. OCT-A was performed before and after a single dialysis session. We performed OCT-A $3 \times 3 \mathrm{~mm}$ and $6 \times 6 \mathrm{~mm}$ scanning area focused on the fovea centralis. Patients were then followed up to 30 days (10 HD sessions) and a total of 73 IDHs were recorded, with 12 patients (60\%) experiencing at least one IDH. Different OCT-A parameters were reduced after dialysis: central choroid thickness (CCT), $6 \times 6 \mathrm{~mm}$ foveal whole vessel density (VD) of superficial capillary plexus (SPC) and $6 \times 6 \mathrm{~mm}$ foveal VD of deep capillary plexus (DCP). At logistic regression analysis, IDH was positively associated with baseline foveal VD of SCP and DCP, while an inverse association was found with the choroid. In Kaplan-Meier analyses of patients categorized according to the ROC-derived optimal thresholds, CCT, the $3 \times 3$ foveal VD of SCP, the $3 \times 3 \mathrm{~mm}$ and $6 \times 6 \mathrm{~mm}$ foveal VD of DCP and the $6 \times 6 \mathrm{~mm}$ foveal VD of SCP were strongly associated with a higher risk of IDH over the 30-days follow-up. In HD patients, a single OCT-A measurement may represent a non-invasive, rapid tool to evaluate the compliance of vascular bed to HD stress and to stratify the risk of IDH in the short term.

Intradialytic hypotension (IDH) is a frequent complication of hemodialysis (HD). It's associated to a high hospitalization rate and to end-organ damage, which is likely mediated through organ ischemia, and consequently to an increased mortality risk. IDH was estimated to affect one third of patients with a higher incidence in subjects with older age, diabetes and cardiovascular comorbidities, longer dialysis vintage, worst nutritional status or higher body mass index ${ }^{1-5}$. The pathogenesis of this condition remains partly unexplained although dysfunctions of the nervous autonomous system ${ }^{6-8}$ and various factors related to the hemodialytic procedure like rapid or excessive ultrafiltration ${ }^{9}$, excessive reduction in osmolality ${ }^{10}$ and reaction to the dialyzer membrane or machine tubing, seem to play a key role. Normal response to hypotension implicates various auto-regulatory mechanisms including the stimulation of sympathetic nervous system aiming at preserving blood flow in vital organs. In other districts, protection mechanisms intervene to maintain adequate blood flow to the metabolic demand of the tissues. This phenomenon can be observed, for instance, in renal arterioles, in coronary circulation or in retinal microcirculation ${ }^{11-13}$. The observation of ocular microcirculation gives us an exceptional chance to directly evaluate in vivo the reactions of human circulation to stress stimuli. Indeed, the ocular microcirculation is involved in systemic disease and early changes in vascular structures may predict the development of systemic vascular disorders ${ }^{14-16}$. Although the study of fundus oculi is mostly employed to assess hypertensive organ damage $\mathrm{e}^{16,17}$, there is now evidence that also systemic hypotension may trigger important vascular changes and even retinal damage as the consequence of local hypoperfusion ${ }^{18,19}$. The effects of hemodialysis on choroid and retinal thickness have already been investigated by structural optical coherence tomography (OCT) ${ }^{20}$ and with the optical coherence tomography angiography (OCT-A $)^{21,22}$, a new method for visualizing the retinal

${ }^{1}$ Renal Unit, University "Magna Graecia" of Catanzaro, Catanzaro, Italy. ${ }^{2}$ Department of Ophthalmology, University Magna Graecia of Catanzaro, Viale Europa, Loc. Germaneto, Catanzaro, Italy. ${ }^{3}$ These authors contributed equally: Giuseppe Coppolino and Adriano Carnevali. ${ }^{\circledR}$ email: adrianocarnevali@live.it 
vasculature and choroidal vascular network that permits a tridimensional evaluation of vascular features such as vessels density. However, to date, no study focused on the possible clinical impact of frequent hypotensive episodes, as those occurring during hemodialysis, on the retinal structures.

In this pilot prospective study, we thus aimed at analyzing possible relationships between various OCT-A metrics and intradialytic hypotension and to evaluate whether OCT-A could represent a useful tool to stratify the hypotensive risk in dialysis patients.

\section{Methods}

Patients selection. Chronic hemodialysis patients from the Dialysis Unit of the University Magna Graecia of Catanzaro, Italy were screened for eligibility to participate into the study. The study was conducted in agreement with the Declaration of Helsinki for research involving human subjects and was approved by the local institutional review board (Comitato Etico Area Centro, Regione Calabria No 38 of 2018). A fully informed consent was obtained from all participants. All subjects were on regular renal replacement treatment with a rhythm of 4-h sessions/three times a week, had a stable dry-weight for at least 3 months before entering the study and had achieved a normotensive edema-free state. Exclusion criteria were dialysis vintage $<6$ months, recent history of hospitalization for cardiovascular diseases, symptomatic pre-dialysis hypotension and severe cognitive or physical impairment ${ }^{3,22}$. Blood pressure (BP) was monitored using an automated sphygmomanometer integrated in the dialysis machine, every $20 \mathrm{~min}$ during and immediately following the dialysis session. Pre-dialysis blood pressure was measured in the non-access arm at the beginning of HD after 10-min rest with the patient seated in the dialysis chair before placement of a dialysis needle. Post-dialysis blood pressure was measured at the end of the session, 10-min after disconnecting the patient from the dialysis circuit. HD modalities were kept constant during the whole study period ${ }^{23}$. All the sessions consisted of standard HD using standard dialysis solutions, with bicarbonate buffer. The dialyzer used was a Flexya dialysis monitor (Bellco, Mirandola, Italy). Adequacy of dialysis was assessed using KT/V, calculated as the natural logarithm of the ratio between initial and final urea concentration. Dialysate sodium concentration was the same for all the patients at $140 \mathrm{meq} / \mathrm{L}$ and dialysate temperature maintained at $36.5^{\circ} \mathrm{C}$. Mean UF rate for hour never exceeded $0.6 \mathrm{~mL} / \mathrm{Kg} / \mathrm{hour}$ with the clinical endpoint to reach an adequate dry weight.

OCT-A metrics. Measurement of OCT-A metrics was performed immediately before and after the first hemodialysis session of the week (Monday or Tuesday) at the Medical Retina \& Imaging Unit of the Department of Ophthalmology, University Magna Graecia of Catanzaro. All measurements were performed by qualified ophthalmologists (A.C. and V.G) experts in retinal imaging, next to the dialysis unit.

Each patient underwent dilated fundus ophthalmoscopy, structural OCT and OCT-A. Ocular exclusion criteria included any other retinal diseases (including retinal vascular diseases, vitreoretinal diseases, history of central serous retinopathy, or macular dystrophies), ocular media opacity, any previous eye surgical intervention or laser photocoagulation in the study eye, poor quality images with significant artefact, inaccurate or incorrect segmentation at the level of the superficial capillary plexus (SCP) and deep capillary plexus (DCP), or subject's inability to abstain from blinking or movement during image acquisition.

OCT and OCT-A image acquisition. OCT-A was performed using XR Avanti AngioVue OCTA (Optovue, Fremont, California, USA). This system uses a split-spectrum amplitude decorrelation angiography (SSADA) algorithm and operates at 70,000 A-scans per second using a light source of $840 \mathrm{~nm}$. SSADA detects variations in reflected OCT signal amplitudes between two consecutive scans ${ }^{24}$. Decorrelation is a mathematical function that quantifies this variation. SSADA splits the OCT signal into different spectral bands, thus increasing the number of usable image frames, in which each undergoes a decorrelation analysis ${ }^{24}$. Blood flowing through vessels causes a change in reflectance over time and results in localized areas of flow decorrelation between frames.

En face images were acquired focusing the fovea centralis (Fig. 1).

Each scan consisted of $304 \times 304$ A-scans with two consecutive B-scans at each fixed position. To reduce motion artifacts, each scan consisted of one orthogonal horizontal and vertical scan ${ }^{24}$. We performed OCT-A $3 \times 3 \mathrm{~mm}$ and $6 \times 6 \mathrm{~mm}$ scanning area focused on the fovea centralis. The instrument software automatically segmented OCT-A scans into four en-face slabs: the SCP, the DCP, the outer retinal plexus and the choriocapillaris plexus. In this study we focus on SCP, DCP plexuses. The software detected the perfused vessel structures within an inner offset at -3 to $-15 \mu \mathrm{m}$ from the inner limiting membrane (ILM) for the evaluation of SCP and within an inner offset at -15 to $-70 \mu \mathrm{m}$ from the ILM for the DCP. The instrument software calculated automatically vessel density (VD) of SCP and DCP in the whole image, foveal and parafoveal zone and it also calculated automatically the foveal avascular zone (FAZ) area expressed in $\mathrm{mm}^{2}$ in the Retina plexus. VD was defined as the percentage of blood flow signal within a defined area. Foveal zone VD was defined as the area with a diameter of $1 \mathrm{~mm}$; parafoveal zone VD was defined as the area with a diameter of $3 \mathrm{~mm}$. For each patient were collected the following parameters: SCP VD and DCP VD of whole image, foveal and parafoveal zone and FAZ for both OCT-A $3 \times 3 \mathrm{~mm}$ and $6 \times 6 \mathrm{~mm}$ scans. Structural OCT was performed using RTVue OCT (Optovue Inc., Fremont, CA, USA), a high-speed and high-resolution spectral domain OCT device with central wavelength of $840 \mathrm{~nm}$, scan rate of 26,000 A-scans/s, and axial resolution of $5 \mu \mathrm{m}$. In this study we were used horinzontal B-scan images centered on the fovea to manually measure central choroidal thickness (CCT) at the fovea: the vertical distance between the hyperscattering retinal pigment epithelium layer and the chorioscleral interface was measured manually using a software caliper built into the custom-made OCT image viewer. The three-dimensional macular scan protocol set to $7 \times 7 \mathrm{~mm}$ containing 101 horizontal line scans each consisting of 513 A scans were used to calculated automatically central retinal thickness (CRT). All measurements were 

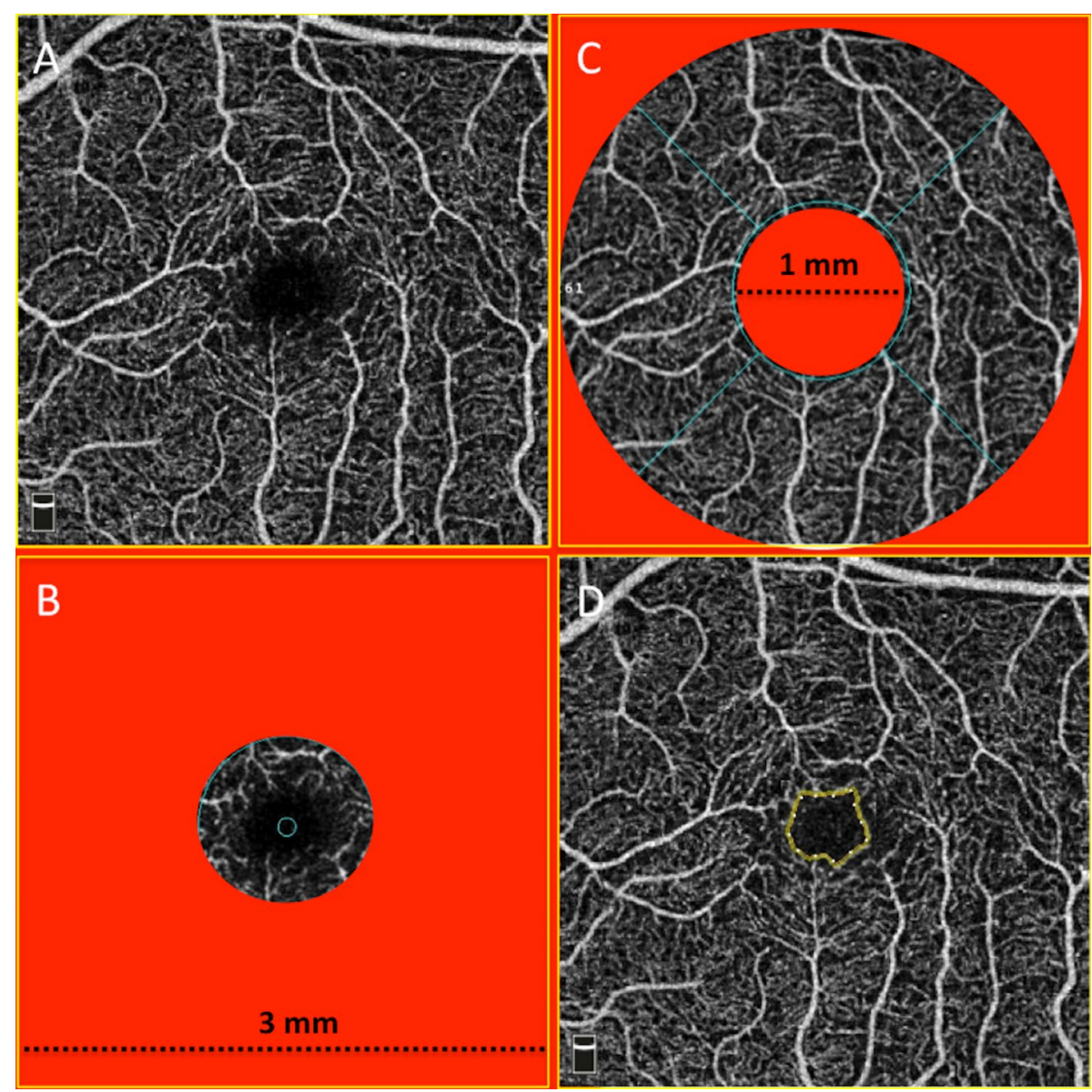

Figure 1. Schematic of optical coherence tomography angiography (OCTA) metrics. (A) OCTA image of whole SCP. (B) OCTA image of foveal region of the SCP delineated at ring of $1 \mathrm{~mm}$. (C) OCTA image of parafoveal region of the SCP delineated between rings of $1 \mathrm{~mm}$ and $3 \mathrm{~mm},((\mathbf{D})$ OCTA image of full retina with the FAZ outlined in yellow.

replicated in both eyes of the same patient and, if successful, a weighted mean of the two values was computed for each parameter and used for analysis.

Prospective follow-up. After the baseline assessment, patients were prospectively followed up to 30 days and any hypotensive episode occurred during the correspondent ten dialysis sessions was recorded. Intradialytic hypotension episodes were defined as systolic blood pressure drop during dialysis greater than $20 \mathrm{mmHg}$ or a decrease in mean arterial pressure of $10 \mathrm{~mm} \mathrm{Hg}$ during dialysis with symptoms such as dizziness, headache, confusion, nausea, sweating requiring nurse intervention considering always a nadir systolic pressure $<90 \mathrm{mmHg}^{25}$.

Statistical analyses. The analyses were performed using the SPSS package (version 24.0; IBM corporation), the MedCalc Statistical Software (version 14.8.1) and the GraphPad prism software (version 8.4.2, San Diego, California USA).

Data were presented as mean \pm SD for normally distributed values (at Kolmogorov-Smirnov test), median [IQ range] for variables with skewed distribution or frequency percentage. Differences between groups were determined by the unpaired T-test for normally distributed values, the Mann-Whitney U test for non-parametric values and the chi-square followed by a Fisher's exact test for frequency distributions. Pre-post dialysis variations of retinal parameters were analyzed by a paired-T-test or by a Wilcoxon signed-rank test for non-parametric values.

Bivariate logistic regression analyses were performed to establish significant predictors of intradialytic hypotension among retinal parameters ${ }^{26}$. In addition, the Pearson $(\mathrm{R})$ correlation was used to test correlations between such parameters and the absolute number of intradialytic hypotension episodes in each patient. A Receiver Operating Characteristics (ROC) analysis was employed to calculate the area under the curve (AUC) for every retinal parameter significantly associated with the outcome at bivariate logistic regression. AUCs were compared by a non-parametric approach. The best cut-off values were computed by the Youden index. Kaplan-Meier curves considering the time to the first intradialytic episode were generated for patients with retinal parameters above or below the optimal, ROC-derived threshold and compared by a Log-Rank test ${ }^{27,28}$. All results were considered significant if the $\mathrm{p}$ value was $\leq 0.05$. 


\begin{abstract}
Results
Study cohort and baseline assessment. The source population consisted of 35 chronic haemodialysis patients. After the initial screening, 15 patients were excluded because refused to participate or due to the presence of significant retinal or ocular alterations hampering the reliability of measurements in both eyes. The final study cohort included 20 prevalent patients with a total of 35 eyes being correctly analyzed. The overall intrapatient concordance of measurements between the two eyes was very high for all parameters ( $\mathrm{R}$ ranging from 0.899 to 0.995$)$. Mean age of patients was $63.7 \pm 11.4$ years and the majority of them were male $(69.2 \%)$. The median dialysis vintage was 25 months (IQR 12-41). Only $20 \%$ of patients were diabetics and the prevalence of cardiovascular comorbidities was relatively low (4.6-33\%). Conversely, almost all patients (89.7\%) were on antihypertensive treatment. Table 1 summarizes the main cohort data.
\end{abstract}

Effects of a single hemodialysis session on OCT-A metrics. All OCT-A metrics were measured before and after a single hemodialysis session. Overall, the majority of parameters remained unchanged at dialysis end, a significant reduction being noticed only for central choroid thickness (median $\Delta$ value 25, IQR 10-57; $\mathrm{p}<0.001$ ), $6 \times 6$ whole VD of SCP (median $\Delta$ value 1.4 , IQR $0.6-3.5 ; \mathrm{p}=0.04$ ) and $6 \times 6$ foveal VD of DCP (median $\Delta$ value 2.5, IQR 0.4-4.6; $\mathrm{p}=0.02$ ) (Fig. 2). Stratified subgroups analyses demonstrated a more prominent reduction in CCT $(\mathrm{p}=0.03)$, as well as in $3 \times 3$ whole VD of SCP and DCP ( $\mathrm{p}=0.05$ for both comparisons) in individuals not experiencing intradialytic hypotensive episodes as compared to others (Table 2).

Prospective follow-up and intradialytic hypotension. During the 30-day follow-up, 73 hypotensive episodes equivalent to an incidence rate of 3.65 episodes/pts/month (95\% CI 2.86-4.58) were registered. All hypotension events were resolved with appropriate therapeutic management and no clinical sequelae. Twelve patients $(60 \%)$ experienced at least one hypotensive episode (mean $6.08 \pm 2.89$ ). At baseline, these individuals were older and had significantly lower systolic and diastolic blood pressure and LDL cholesterol but higher serum ferritin levels as compared with individuals without hypotensive episodes. With respect to retinal parameters, they displayed a statistically thinner choroid $(\mathrm{p}=0.04)$ and a reduced $6 \times 6$ FAZ $(\mathrm{p}=0.03)$ but a larger $3 \times 3$ and $6 \times 6$ foveal VD of SCP ( $p=0.04$ and 0.002 , respectively), as well as a larger $3 \times 3$ and $6 \times 6$ foveal VD of DCP $(\mathrm{p}=0.02$ and 0.04 , respectively). No statistical differences were noticed with respect to other parameters. Table 1 resumes the main characteristics of the two study subgroups.

Associations between OCT-A metrics and IDH. Pre-dialysis OCT-A metrics were tested separately into a bivariate logistic regression analysis to find possible associations with the occurrence of intradialytic hypotension. As summarized in Table 3, a statistically significant positive association was found with the $3 \times 3$ and $6 \times 6$ foveal VD of SCP (OR 1.122; 95\% CI 1.001-1.276; $p=0.05$ and OR 1.143; 95\% CI 1.034-1.264; $p=0.009$, respectively) and the $3 \times 3$ and $6 \times 6$ foveal VD of DCP (OR 1.130; 95\% CI 1.011-1.263; $\mathrm{p}=0.03$ and OR 1.072; 95\% CI 1.002-1.157; $\mathrm{p}=0.04$, respectively), while an inverse association was found with the CCTOR 0.990; 95\% CI 0.979-0.999; $\mathrm{p}=0.05)$. In addition, CRT and the $3 \times 3$ and $6 \times 6$ foveal VD of SCP and DCP were directly associated to the absolute number of hypotensive episodes ( $R$ spanning from 0.342 to 0.557 ), while the $6 \times 6$ FAZ was negatively correlated $(\mathrm{R}=-0.374, \mathrm{p}=0.02$; Fig. 3 , Table 4$)$.

Predictive value of OCT-A metrics on IDH. The diagnostic capacity to identify patients with intradialytic hypotension episodes was tested by ROC analyses for each parameter significantly associated with the outcome at logistic regression. The AUC for $3 \times 3$ foveal VD of SCP was statistically not significant. Conversely, the AUCs of CCT, $3 \times 3$ foveal VD of DCP and $6 \times 6$ foveal VD of SCP and DCP were all significant (range 0.704 to 0.783 ; p ranging from 0.03 to 0.006 ), although not different from each other (Fig. 4 ). Table 5 provides a summary of ROC analyses of retinal parameters, including the best cut-off values computed for each variable able to discriminate patients with or without the outcome of interest.

Kaplan-Meier curves were thus generated for patients categorized according to such ROC-derived optimal thresholds (Fig. 5). All the parameters tested in these analyses, namely the CCT, the $3 \times 3$ foveal VD of SCP, the $3 \times 3$ and $6 \times 6$ foveal VD of DCP and the $6 \times 6$ foveal VD of SCP, resulted strongly associated with a higher risk of intradialytic hypotension (Log-rank test ranging from 7.78 to 12.41) over the 30-days follow-up.

\title{
Discussion
}

In our study, we demonstrated that chronic hemodialysis patients experiencing frequent IDH episodes showed a different ocular pattern with respect to those not experiencing this complication. Furthermore, a simple measurement of retinal and choroid parameters by OCT-A before a single dialysis session may help predicting the risk of following IDH in the short-term.

Currently, OCT-A and analytic softwares have facilitated automated and manual processing of macular perfusion data ${ }^{29-31}$. The effect of hemodialysis on perfused vessel density is still debated, with some studies reporting a significant reduction ${ }^{21,32}$ and some others indicating no differences ${ }^{33}$ or even a paradoxical increase ${ }^{34}$. In our study, we reported a barely or not significant drop of vessels dimension or density in choroidal and retinal analysis in the whole study population while, interestingly, a more prominent decrease in central choroid thickness and whole SCP and DCP were noticed between patients experiencing IDHs or not. Similar changes previously described in other studies employing OCT-A in HD patients were referred to a decrease in body weight, systolic blood pressure and serum osmolarity ${ }^{35}$. Blood pressure in patients on hemodialysis routinely exhibits marked variability, tending to be highest in the immediate pre-dialysis period and decreasing during the intradialytic period. The majority of patients experience an overall decline in BP, on average in the range of 30-40 $\mathrm{mmHg}$, 


\begin{tabular}{|c|c|c|c|c|}
\hline & $\begin{array}{l}\text { All } \\
\mathrm{N}=\mathbf{2 0}\end{array}$ & $\begin{array}{l}\text { Hypotension } \\
\mathrm{N}=12\end{array}$ & $\begin{array}{l}\text { No-hypotension } \\
\mathrm{N}=8\end{array}$ & $p$ \\
\hline Age (years) & $63.7 \pm 11.4$ & $68.2 \pm 11.3$ & $57.4 \pm 8.4$ & 0.02 \\
\hline Gender (\%Male) & 69.2 & 65.5 & 62.5 & 0.44 \\
\hline Dry weight (kg) & $66.2 \pm 14.3$ & $63.8 \pm 11.2$ & $69.7 \pm 17.6$ & 0.21 \\
\hline $\mathrm{Kt} / \mathrm{V}$ & $1.37 \pm 0.15$ & $1.4 \pm 0.14$ & $1.3 \pm 0.12$ & 0.18 \\
\hline Dialysis vintage (mo.) & $25[12-41]$ & $25[13-36]$ & $25[10-40]$ & 0.81 \\
\hline Diabetes (\%) & 20.5 & 22.5 & 17.5 & 0.55 \\
\hline Past smokers (\%) & 30.3 & 29.3 & 30.1 & 0.57 \\
\hline History of myocardial ischemia (\%) & 33.3 & 35 & 25 & 0.64 \\
\hline Peripheral vasculopathy (\%) & 10.3 & 10.5 & 9.3 & 0.50 \\
\hline Cerebrovascular disease (\%) & 5.1 & 4.6 & 6.8 & 0.70 \\
\hline Hypertension (\%) & 89.7 & 75.4 & 100 & 0.08 \\
\hline Systolic blood pressure (mmHg) & $136.4 \pm 8.0$ & $139.8 \pm 6.1$ & $143.1 \pm 5.1$ & 0.10 \\
\hline Diastolic blood pressure (mmHg) & $71.5 \pm 6.8$ & $69.6 \pm 7.3$ & $74.4 \pm 4.9$ & 0.06 \\
\hline Serum phosphate $(\mathrm{mg} / \mathrm{dL})$ & $5.8 \pm 0.77$ & $5.7 \pm 0.9$ & $5.9 \pm 0.5$ & 0.51 \\
\hline Serum calcium $(\mathrm{mg} / \mathrm{dL})$ & $8.6 \pm 0.73$ & $8.6 \pm 0.5$ & $8.8 \pm 0.9$ & 0.39 \\
\hline Parathormone (pg/mL) & $318[245-476]$ & $366[285-576]$ & 298 [199-386] & 0.17 \\
\hline Albumin $(\mathrm{g} / \mathrm{dL})$ & $4.3 \pm 0.9$ & $4.4 \pm 0.6$ & $4.1 \pm 0.4$ & 0.65 \\
\hline LDL cholesterol (mg)/dL) & $96.2 \pm 31.3$ & $88.1 \pm 29.1$ & $107.7 \pm 31.7$ & 0.05 \\
\hline Total cholesterol (mg/dL) & $222.4 \pm 31.6$ & $225.0 \pm 31.1$ & $218.7 \pm 32.9$ & 0.55 \\
\hline Triglycerides $(\mathrm{mg} / \mathrm{dL})$ & $149.9 \pm 48.0$ & $144.8 \pm 44.9$ & $157.2 \pm 52.7$ & 0.43 \\
\hline Hematocrit (\%) & $34.5 \pm 3.6$ & $34.2 \pm 2.7$ & $34.8 \pm 4.7$ & 0.63 \\
\hline Hemoglobin $(\mathrm{g} / \mathrm{dL})$ & $10.8 \pm 0.9$ & $10.7 \pm 0.77$ & $10.8 \pm 1.17$ & 0.94 \\
\hline White blood cells $\left(\mathrm{n} \times 10^{3}\right)$ & $6.6 \pm 1.5$ & $6.4 \pm 1.6$ & $6.9 \pm 1.4$ & 0.24 \\
\hline Uric acid $(\mathrm{mg} / \mathrm{dL})$ & $5.9 \pm 1.0$ & $5.9 \pm 0.76$ & $6.1 \pm 1.4$ & 0.59 \\
\hline C-reactive protein $(\mathrm{mg} / \mathrm{L})$ & $3.7[3.2-5.7]$ & $3.9[2.5-6.7]$ & $2.7[1.2-6.4]$ & 0.35 \\
\hline Ferritin $(\mathrm{mg} / \mathrm{dL})$ & $306[219-370]$ & $378[306-927]$ & 232 [151-289] & 0.01 \\
\hline Serum iron $(\mathrm{mg} / \mathrm{dL})$ & $72.6 \pm 36.8$ & $77.1 \pm 40.6$ & $66.2 \pm 30.6$ & 0.37 \\
\hline b2-microglobulin (mg/L) & $26.2 \pm .6 .2$ & $25.9 \pm 7.1$ & $26.6 \pm 4.7$ & 0.76 \\
\hline Urea (mg/dL) & $142.9 \pm 31.6$ & $143.2 \pm 34.6$ & $142.5 \pm 27.7$ & 0.94 \\
\hline Fibrinogen $(\mathrm{mg} / \mathrm{dL})$ & $331.4 \pm 97.1$ & $353.4 \pm 86.8$ & $299.6 \pm 104.8$ & 0.08 \\
\hline \multicolumn{5}{|l|}{ Ocular parameters } \\
\hline CRT & $276.7 \pm 25.9$ & $296.3 \pm 118$ & $251.8 \pm 23.1$ & 0.14 \\
\hline Choroid central thickness & $300.8 \pm 73.9$ & $280.4 \pm 72.6$ & $330.1 \pm 65.3$ & 0.04 \\
\hline FAZ-SCP $3 \times 3 \mathrm{~mm}$ & $0.24[0.16-0.34]$ & $0.22[0.16-0.26]$ & $0.24[0.16-0.39]$ & 0.28 \\
\hline FAZ-SCP $6 \times 6 \mathrm{~mm}$ & $0.24[0.15-0.30]$ & $0.21[0.15-0.26]$ & $0.27[0.18-0.39]$ & 0.03 \\
\hline WHOLE-SCP $3 \times 3 \mathrm{~mm}$ & $41.5 \pm 3$ & $41.5 \pm 4.4$ & $42.3 \pm 4$ & 0.56 \\
\hline WHOLE-SCP $6 \times 6 \mathrm{~mm}$ & $47.3 \pm 3.7$ & $46.3 \pm 5$ & $47.3 \pm 4.6$ & 0.54 \\
\hline WHOLE-DCP $3 \times 3 \mathrm{~mm}$ & $47.8 \pm 4.3$ & $46.9 \pm 5.4$ & $47.8 \pm 4.5$ & 0.61 \\
\hline WHOLE-DCP $6 \times 6 \mathrm{~mm}$ & $44.2 \pm 7$ & $44.5 \pm 5.8$ & $44.6 \pm 5.9$ & 0.96 \\
\hline PARAFOVEA-SCP $3 \times 3 \mathrm{~mm}$ & $43 \pm 7.5$ & $44 \pm 4.6$ & $45.4 \pm 4.1$ & 0.34 \\
\hline PARAFOVEA-SCP $6 \times 6 \mathrm{~mm}$ & $48.1 \pm 3.9$ & $47.2 \pm 4.6$ & $48.8 \pm 4.7$ & 0.32 \\
\hline PARAFOVEA-DCP $3 \times 3 \mathrm{~mm}$ & $49.7 \pm 4.7$ & $48.4 \pm 5.8$ & $50.2 \pm 4.7$ & 0.32 \\
\hline PARAFOVEA-DCP6×6 mm & $50.1 \pm 6.7$ & $50.1 \pm 4.3$ & $50.4 \pm 7.2$ & 0.86 \\
\hline FOVEA-SCP $3 \times 3 \mathrm{~mm}$ & $20.7 \pm 12$ & $19.9 \pm 3.3$ & $16 \pm 7.9$ & 0.04 \\
\hline FOVEA-SCP $6 \times 6 \mathrm{~mm}$ & $23.8 \pm 9.3$ & $24.7 \pm 8.2$ & $15.6 \pm 8.1$ & 0.002 \\
\hline FOVEA-DCP $3 \times 3 \mathrm{~mm}$ & $35.8 \pm 8.9$ & $37.1 \pm 5.8$ & $31.4 \pm 8.1$ & 0.02 \\
\hline FOVEA-DCP $6 \times 6 \mathrm{~mm}$ & $39.4 \pm 11$ & $41.2 \pm 9.9$ & $34.8 \pm 9.7$ & 0.04 \\
\hline
\end{tabular}

Table 1. Main laboratory and clinical characteristics of the whole study population and differences between subgroups. CRT central retinal thickness, FAZ foveal avascular zone, DCP deep capillary plexus, SCP superficial capillary plexus. 

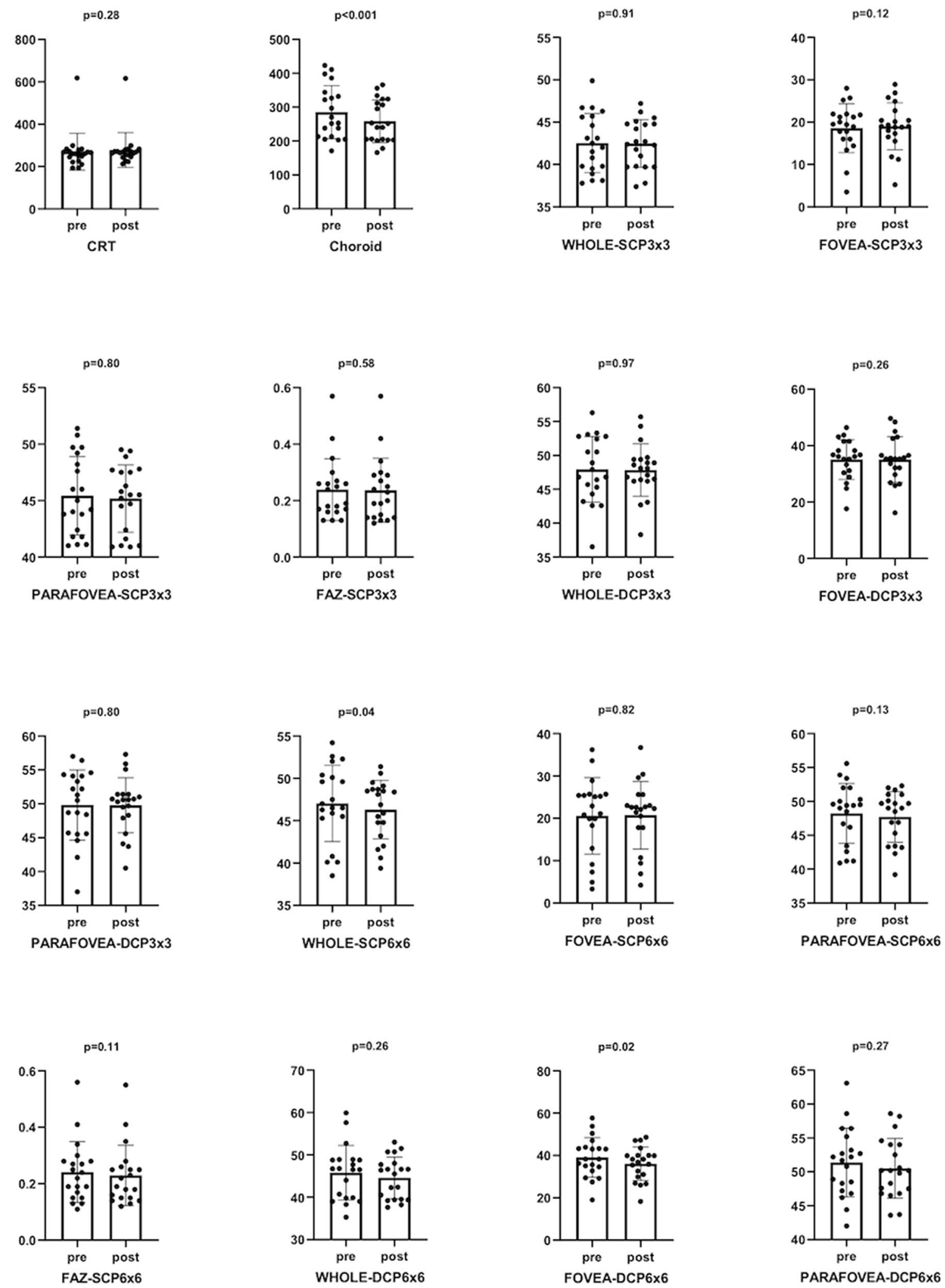

Figure 2. Pre-post dialysis variations in OCT-A metrics. A more prominent significant drop was found in choroidal parameters (Choroid $\mathrm{p}<0.001$, WHOLE-SCP6X6 and FOVEA-DCP6X6 $\mathrm{p}<0.05$ ) respect to retinal parameters. 


\begin{tabular}{|l|c|c|c|l|}
\hline & $\begin{array}{l}\text { All } \\
\mathbf{N = 2 0}\end{array}$ & $\begin{array}{l}\text { Hypotension } \\
\mathbf{N = 1 2}\end{array}$ & $\begin{array}{l}\text { No-hypotension } \\
\mathbf{N}=\mathbf{8}\end{array}$ & $\boldsymbol{p}$ \\
\hline$\Delta$ CRT & $0[-3$ to 2$]$ & $-2[-3$ to 2$]$ & $1[-1.7$ to 2$]$ & 0.21 \\
\hline$\Delta$ Choroid central thickness & $25[10$ to 57$]$ & $\mathbf{2 0}[\mathbf{2}$ to 45$]$ & $\mathbf{3 8}[\mathbf{2 1}$ to $6 \mathbf{9 . 7}]$ & $\mathbf{0 . 0 3}$ \\
\hline$\Delta$ FAZ-SCP $3 \times 3 \mathrm{~mm}$ & $0[-0.01$ to 0.01$]$ & $0[-0.1$ to 0.1$]$ & $0.01[-0.04$ to 0.01$]$ & 0.17 \\
\hline$\Delta$ FAZ-SCP $6 \times 6 \mathrm{~mm}$ & $0.05[-0.04$ to 0.01$]$ & $0.03[-0.02$ to 0.01$]$ & $0.07[-0.02$ to 0.04$]$ & 0.16 \\
\hline$\Delta$ WHOLE-SCP $3 \times 3 \mathrm{~mm}$ & $0.3[-1.6$ to 1.7$]$ & $\mathbf{0 . 1}[-\mathbf{2 . 7}$ to $\mathbf{1 . 5}]$ & $\mathbf{1}[-\mathbf{0 . 6}$ to $2 . \mathbf{4}]$ & $\mathbf{0 . 0 5}$ \\
\hline$\Delta$ WHOLE-SCP $6 \times 6 \mathrm{~mm}$ & $1.4[0.6$ to 3.5$]$ & $1.7[0.5$ to 3.6$]$ & $1.2[0.6$ to 3.2$]$ & 0.84 \\
\hline$\Delta$ WHOLE-DCP $3 \times 3 \mathrm{~mm}$ & $-0.1[-3.2$ to 1.2$]$ & $-\mathbf{1 . 3}[-\mathbf{3 . 3}$ to $\mathbf{1 . 1}]$ & $\mathbf{0 . 3}[-\mathbf{0 . 8}$ to 2.3$]$ & $\mathbf{0 . 0 5}$ \\
\hline$\Delta$ WHOLE-DCP $6 \times 6 \mathrm{~mm}$ & $-0.6[-2.6$ to 3.5$]$ & $-0.6[-3.5$ to 3.5$]$ & $0.05[-2.3$ to 6.6$]$ & 0.50 \\
\hline$\Delta$ PARAFOVEA-SCP $3 \times 3 \mathrm{~mm}$ & $0.5[-1.6$ to 1.6$]$ & $0.5[-2.8$ to 1.3$]$ & $1.05[-0.1$ to 2.2$]$ & 0.17 \\
\hline$\Delta$ PARAFOVEA-SCP $6 \times 6 \mathrm{~mm}$ & $1[-0.8$ to 3.3$]$ & $2.3[-2.9$ to 4.4$]$ & $0.2[-0.6$ to 2.6$]$ & 0.20 \\
\hline$\Delta$ PARAFOVEA-DCP $3 \times 3 \mathrm{~mm}$ & $0.3[-3$ to 1.9$]$ & $-1.9[-3$ to 1.5$]$ & $0.9[-2.1$ to 4.2$]$ & 0.17 \\
\hline$\Delta$ PARAFOVEA-DCP $6 \times 6 \mathrm{~mm}$ & $-1.3[-3$ to 4.7$]$ & $-1.3[-1.8$ to 4.7$]$ & $-0.9[-4.8$ to 5.6$]$ & 0.76 \\
\hline$\Delta$ FOVEA-SCP $3 \times 3 \mathrm{~mm}$ & $-0.5[-1.7$ to 1.5$]$ & $0.5[-2.8$ to 1.3$]$ & $-0.5[-2.6$ to 1.1$]$ & 0.76 \\
\hline$\Delta$ FOVEA-SCP $6 \times 6 \mathrm{~mm}$ & $0.5[-1$ to 2.6$]$ & $2.4[-0.5$ to 3$]$ & $0.2[-0.6$ to 2.6$]$ & 0.21 \\
\hline$\Delta$ FOVEA-DCP $3 \times 3 \mathrm{~mm}$ & $-0.1[-2.5$ to 1.4$]$ & $-0.1[-3.5$ to 1.7$]$ & $-0.1[-2.2$ to 1.4$]$ & 0.89 \\
\hline$\Delta$ FOVEA-DCP $6 \times 6 \mathrm{~mm}$ & $2.5[0.4$ to 4.6$]$ & $-1.3[-1.5$ to 2$]$ & $-0.9[-4.8$ to 5.6$]$ & 0.38 \\
\hline
\end{tabular}

Table 2. Pre-post dialysis change $(\Delta)$ in OCT-A metrics in the whole cohort and in subgroups. CRT central retinal thickness, FAZ foveal avascular zone, DCP deep capillary plexus, SCP superficial capillary plexus. Statistically significant differences between subgroups are shown in bold.

\begin{tabular}{|l|l|l|l|}
\hline Parameter & OR & $\mathbf{9 5 \%}$ CI & $\mathbf{p}$ \\
\hline Choroid central thickness & 0.990 & $0.979-0.999$ & 0.05 \\
\hline FOVEA-SCP $3 \times 3 \mathrm{~mm}$ & 1.122 & $1.001-1.276$ & 0.05 \\
\hline FOVEA-DCP $3 \times 3 \mathrm{~mm}$ & 1.130 & $1.011-1.263$ & 0.03 \\
\hline FOVEA-SCP $6 \times 6 \mathrm{~mm}$ & 1.143 & $1.034-1.264$ & 0.009 \\
\hline FOVEA-DCP $6 \times 6 \mathrm{~mm}$ & 1.072 & $1.002-1.157$ & 0.04 \\
\hline
\end{tabular}

Table 3. OCT-A parameters significantly associated to the occurrence of intradialytic hypotension at logistic regression analyses. $C R T$ central retinal thickness, $F A Z$ foveal avascular zone, DCP deep capillary plexus, SCP superficial capillary plexus.

which was in line with the findings in our cohort. Such a typical trend of BP occurs without adverse symptoms, being well tolerated by patients. Occasionally hypotension could occur if fluid removal during hemodialysis exceeds the correct evaluated ultrafiltration rate. Patients in our analysis were stringently enrolled avoiding excessive or rapid fluid removal during observational period. Nevertheless, in line with common clinical practice in some subjects we recorded hypotensive events. OCT-A analysis in the IDH group with respect to patients not experiencing this complication showed a lower size in choroid at basal measurement and small changes after HD. Notoriously, the choroidal circulation has the highest blood supply per organ area and has a specific extrinsic autonomic regulation unlike retinal vessels ${ }^{36}$. Sympathetic innervation includes noradrenergic and neuropeptide fibers while parasympathetic innervation consists of cholinergic fibers ${ }^{37,38}$. Decrease in choroidal flow is mediated by sympathetic innervation through release of noradrenaline ${ }^{6}$ while an increase activates parasympathetic reflexes by nitric oxide release ${ }^{34}$. In dialysis patients, the particular response pattern of ocular vessels observed in the IDH group could reflect a more pronounced, overall dysautonomic state, a pathological condition that is a hallmark of uremia. Patients prone to IDH are known to have an amplified sensitivity to the Bezold-Jarisch reflex. This reflex begins when myocardial mechanoreceptors are activated in response to ventricular under filling and leads to vagal afferent inhibition of the medullary cardiovascular center. Consequently, the patient experiences a dramatic decrease in sympathetic nervous system activity with following arteriolar vasodilation, bradycardia, and IDH. This state is probably a consequence of uremic toxins interacting with the neural termination and affecting their activation through superoxide-dependent effects on neural activity in particular by reducing nitric oxide (NO) bioavailability and inducing systemic inflammation ${ }^{39}$. In IDH patients, the response to hypotension in single vascular districts aims at re-establishing a stable blood volume after fluid excess removal but these mechanisms are often inadequate. Interestingly, pre-dialysis choroid thickness showed an impressive ability to predict the occurrence of IDH, as showed by both diagnostic and survival analyses. Dysregulation of ocular blood flow was deeply studied and observed in diabetic retinopathy with significant neuronal and glial changes leading to a reduction in the functional hyperemia response and in basal blood flow ${ }^{40}$. Our findings could therefore be interpreted as a typical morphological adaptation of vascular bed in certain patients, suggesting that OCT-A could represent a surrogate, non-invasive way to evaluate autonomic vasomotor function in dialysis patients. 


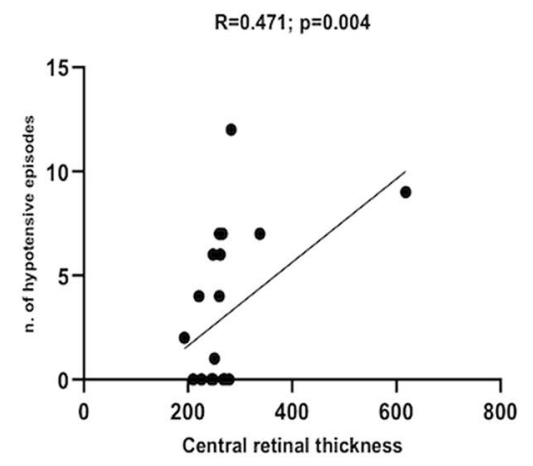

$R=0.557 ; p=0.001$

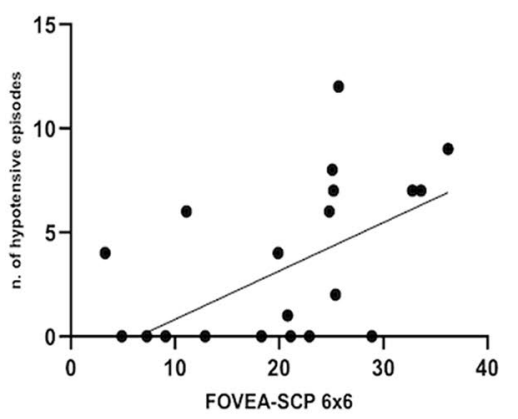

$R=0.342 ; p=0.04$

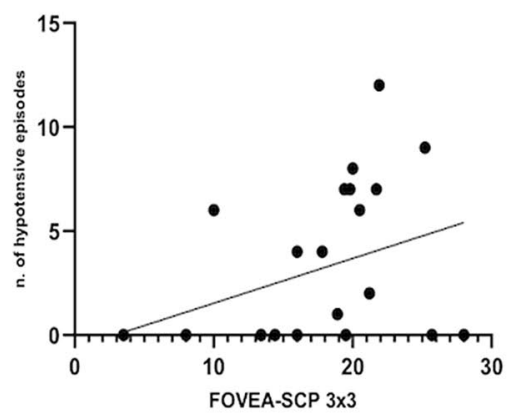

$R=0.417 ; p=0.01$

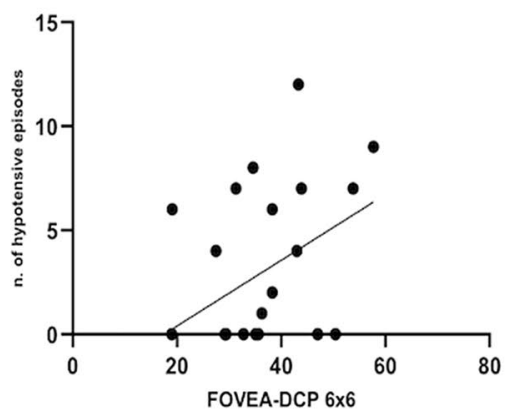

$R=0.438 ; p=0.008$

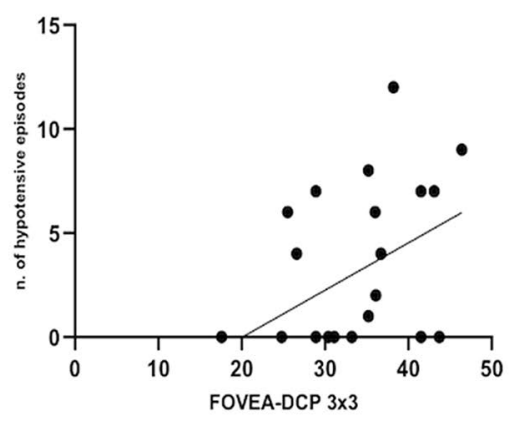

$R=-0.374 ; p=0.02$

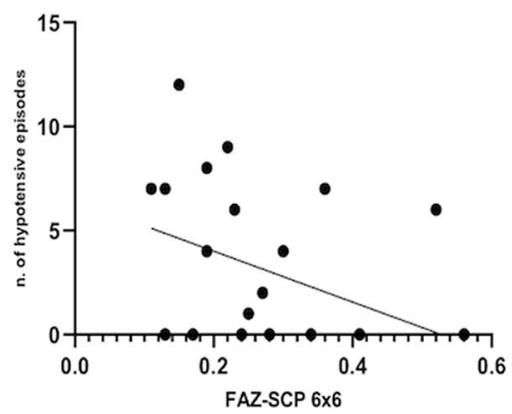

Figure 3. Correlations between number of hypotensive episodes and OCT-A parameters.

\begin{tabular}{|l|l|l|}
\hline Parameter & R & p \\
\hline CRT & 0.471 & 0.004 \\
\hline FOVEA-SCP $3 \times 3 \mathrm{~mm}$ & 0.342 & 0.04 \\
\hline FOVEA-DCP $3 \times 3 \mathrm{~mm}$ & 0.438 & 0.008 \\
\hline FOVEA-SCP $6 \times 6 \mathrm{~mm}$ & 0.557 & 0.001 \\
\hline FOVEA-DCP $6 \times 6 \mathrm{~mm}$ & 0.417 & 0.01 \\
\hline FAZ-SCP $6 \times 6 \mathrm{~mm}$ & -0.374 & 0.02 \\
\hline
\end{tabular}

Table 4. Significant associations (Pearson coefficient) between OCT-A parameters and the number of hypotensive episodes during follow-up. CRT central retinal thickness, FAZ foveal avascular zone, DCP deep capillary plexus, $S C P$ superficial capillary plexus.

In our analysis, also VD of DCP and SCP plexuses in foveal shots predicted the occurrence of hypotensive episodes.

Differently from choroid, retinal circulation is similar to brain circulation but lacks of autonomic innervation. The capillary unit consists of a continuous endothelium and intramural pericytes. Structural retinal changes are known as early indicators of the presence and severity of coronary artery disease ${ }^{37}$, also predicting cardiovascular mortality ${ }^{41,42}$. Differences in retinal vessels density in the IDH group could also reflect endothelial dysfunction. In normal subjects the reduction of systemic blood pressure results in the constriction of pericytes and reduction in blood flow to choroidal and retinal capillaries mediated by oxidization and nitration stimuli ${ }^{43-46}$. Reduction in ocular blood pressure causes the release of vasodilator factors such as adenosine ${ }^{47}$ and N-methyl-D aspartic $\operatorname{acid}^{48}$.

In a healthy animal model, Tami et al. ${ }^{49}$ recently showed that a drop of ocular perfusion pressure under $80 \mathrm{mmHg}$, consequent to systemic hypotension, activates vasodilatory mechanisms able to maintain retinal blood 


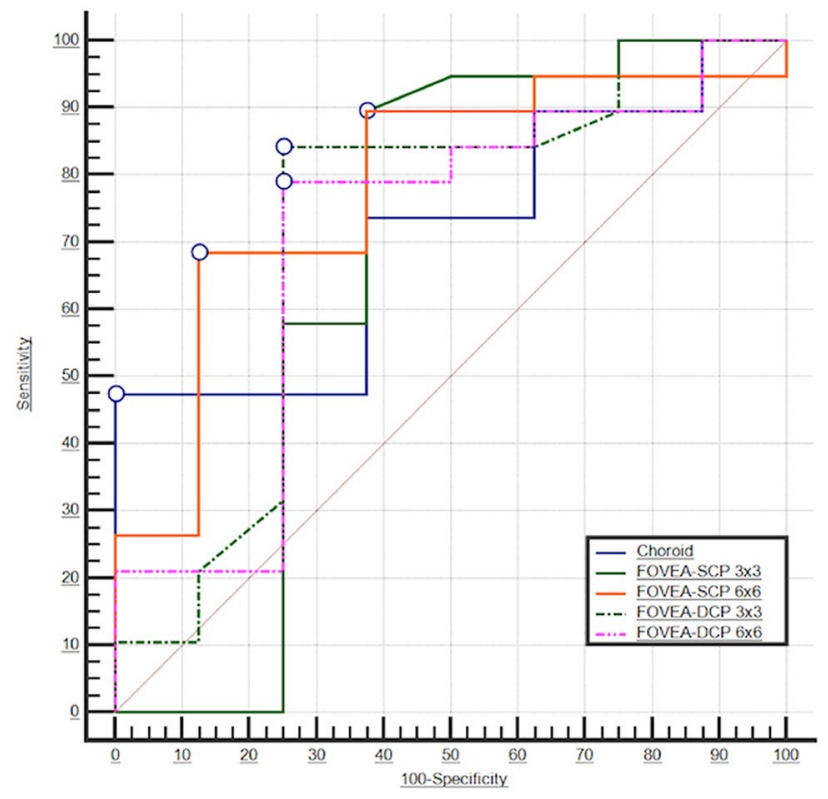

Figure 4. Areas under the curve (AUCs) of OCT-A parameters to identify HD patients experiencing hypotensive episodes. White circles indicate optimal thresholds (Youden index) for each variable. Differences in AUCs were statistically not significant.

\begin{tabular}{|c|c|c|c|c|c|}
\hline & AUC [95\% CI] & $\mathbf{p}$ & Best cut-off & Sens.\% & Spec. $\%$ \\
\hline Choroid central thickness & $0.711[0.535-0.886]$ & 0.01 & $\leq 242$ & $47.3[24.4-71.1]$ & $100[79.4-100]$ \\
\hline FOVEA-DCP $3 \times 3 \mathrm{~mm}$ & $0.720[0.532-0.908]$ & 0.02 & $>33.2$ & $84.2[60.4-96.6]$ & $75[47.6-92.7]$ \\
\hline FOVEA-SCP $6 \times 6 \mathrm{~mm}$ & $0.783[0.622-0.944]$ & 0.006 & $>22.9$ & $68.4[43.4-87.4]$ & $87.5[61.7-98.4]$ \\
\hline FOVEA-DCP $6 \times 6 \mathrm{~mm}$ & $0.704[0.516-0.892]$ & 0.03 & $>35.6$ & 78.9 [54.4-93.9] & $75[47.6-92.7]$ \\
\hline FOVEA-SCP $3 \times 3 \mathrm{~mm}$ & $0.674[0.460-0.889]$ & 0.11 & $>16$ & 89.4 [66.9-98.7] & $62.5[35.4-84.8]$ \\
\hline
\end{tabular}

Table 5. Areas under the curve (AUCs) and best cut-off values (Youden index) of OCT-A parameters to detect patients with intradialytic hypotensive episodes. FAZ foveal avascular zone, SCP superficial capillary plexus. Statistically significant AUCs are highlighted in bold.

flow. Feedback autoregulation was blunted by the administration of L-NAME (N omega-nitro-L-arginine methyl ester) a well-known inhibitor of nitric oxide synthase (NOS) used to show endothelium-dependent relaxations of arteries ${ }^{50}$. Maintenance of blood flow patency is age-related ${ }^{51}$. In this context, uremia was often considered a model of accelerated aging ${ }^{52}$ and serious vascular dysfunction. Exposure to the uremic toxic milieu and repetitive episodes of hypotension as observed in some HD patients could also lead to loss of this regulation system ${ }^{53}$.

Our study has some strengths and limits that deserve mentioning. The main strength was the homogeneity of cohort, the sequential pre-post dialysis evaluation of retinal parameters and a prospective phase that was long enough to capture a sufficient number of IDH episodes according to validated criteria. The main limit is probably represented by the small, single-centre evaluation and the lack of information on markers able to demonstrate dysregulation of autonomic nervous system. However, despite the limited number of cases analyzed, we reported a significant number of patients experiencing IDHs and a quite high absolute number of episodes over the follow-up period, which allowed to uncover significant associations between retinal parameters and hypotensive episodes at both logistic and survival analyses and to define a strong diagnostic capacity at ROC analyses for some of these parameters. In conclusion, in this pilot study, we found OCT-A as a potential tool to screen and measure the compliance of vascular bed to hemodialytic stress. Larger investigations are needed to confirm whether this approach may represent also an alternative way to stratify patients according to the risk of developing dangerous hypotensive complications in the short-mid-term. 
A

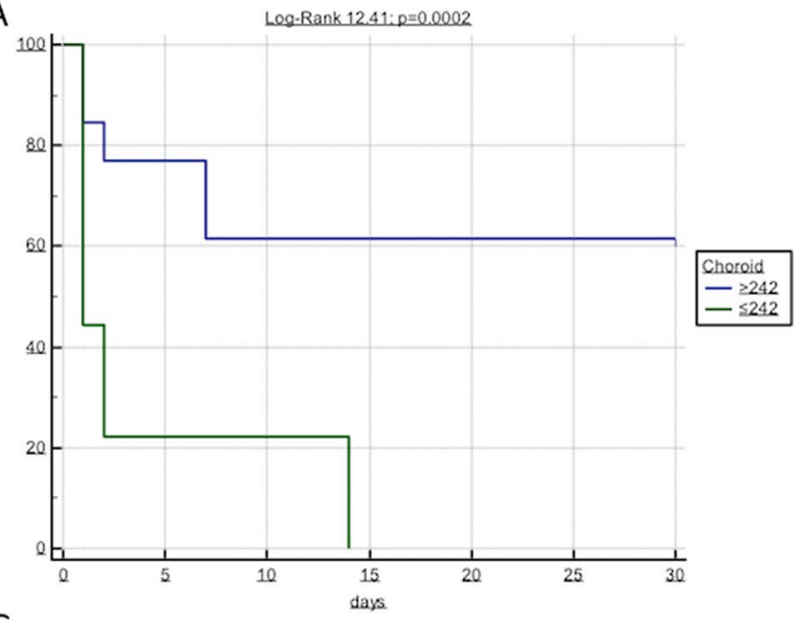

C

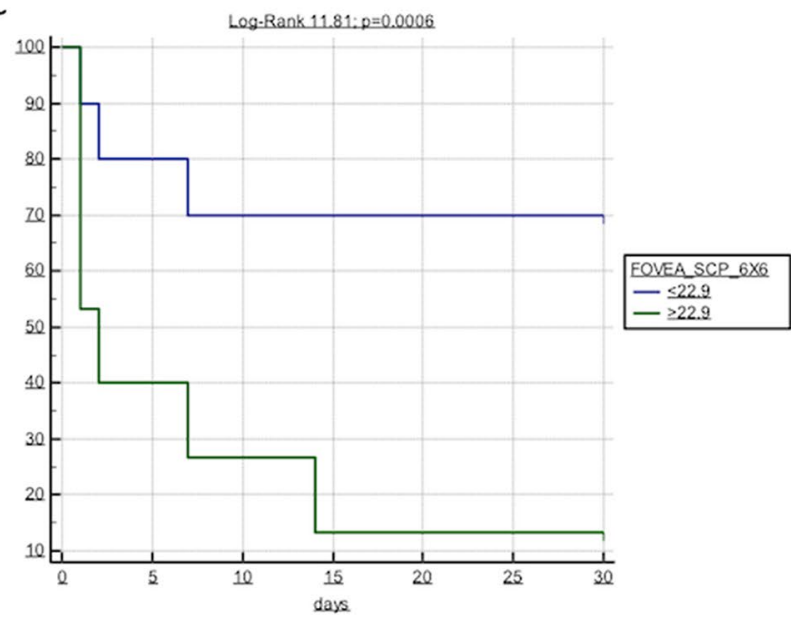

B

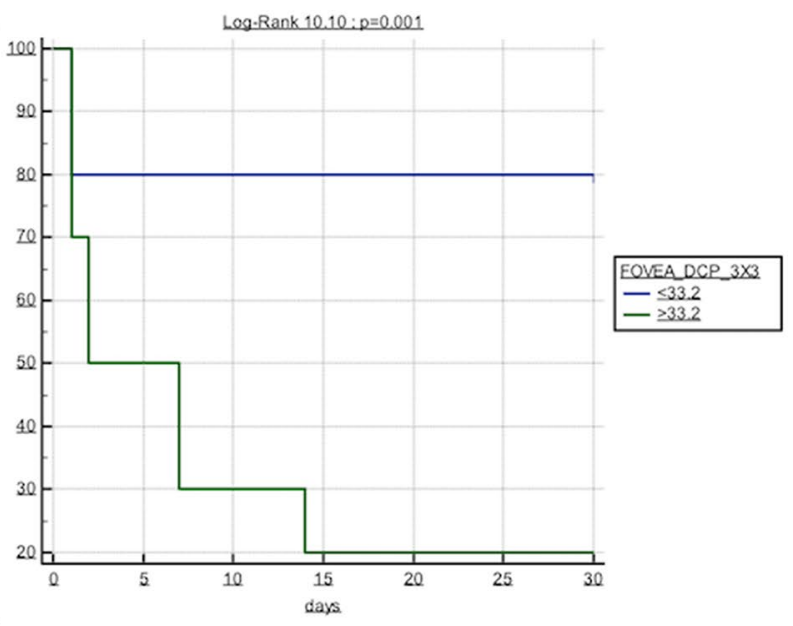

D

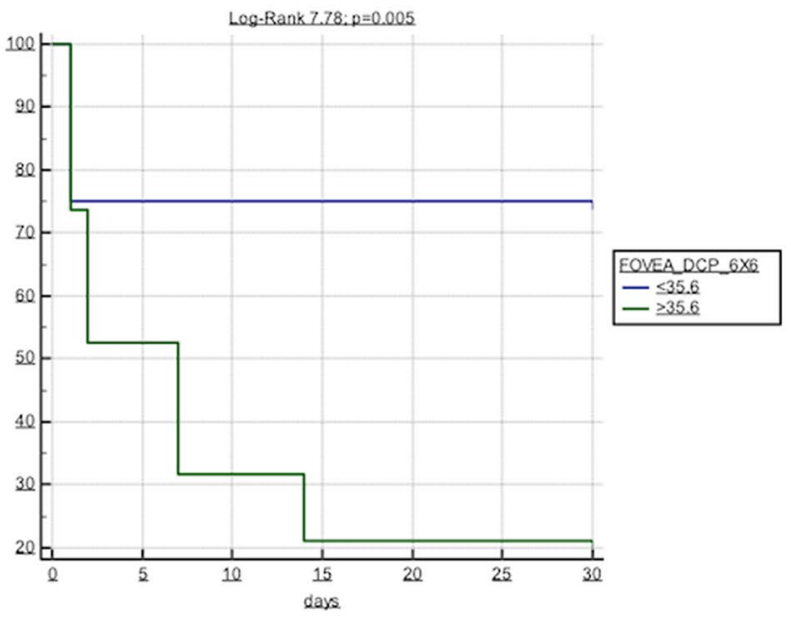

Figure 5. Kaplan-Meier survival curves of intradialytic hypertension-free patients according to the optimal ROC-derived cut-off for (A) Choroid, (B) $3 \times 3$ foveal DCP, (C) $6 \times 6$ foveal SCP and (D) $6 \times 6$ foveal DCP.

Received: 4 November 2020; Accepted: 12 March 2021

Published online: 30 March 2021

\section{References}

1. Stefansson, B. V. et al. Intradialytic hypotension and risk of cardiovascular disease. Clin. J. Am. Soc. Nephrol. 9, 2124-2132 (2014).

2. Flythe, J. E., Xue, H., Lynch, K. E., Curhan, G. C. \& Brunelli, S. M. Association of mortality risk with various definitions of intradialytic hypotension. J. Am. Soc. Nephrol. 26, 724-734 (2015).

3. Sands, J. J. et al. Intradialytic hypotension: Frequency, sources of variation and correlation with clinical outcome. Hemodial. Int. 18, 415-422 (2014).

4. Provenzano, M. et al. Epidemiology of cardiovascular risk in chronic kidney disease patients: The real silent killer. Articolo Riv. 20, 209-220 (2019).

5. Coppolino, G. et al. Exploring the effects of DPP-4 inhibitors on the kidney from the bench to clinical trials. Pharmacol. Res. 129, 274-294 (2018).

6. Robinson, T. G. \& Carr, S. J. Cardiovascular autonomic dysfunction in uremia. Kidney Int. 62, 1921-1932 (2002).

7. Coppolino, G., Lucisano, G., Bolignano, D. \& Buemi, M. Acute cardiovascular complications of hemodialysis. Minerva Urol. Nefrol. 62, 67-80 (2010).

8. Provenzano, M. et al. Unraveling cardiovascular risk in renal patients: A new take on old tale. Front. Cell Dev. Biol. 7, 314 (2019).

9. Santoro, A. et al. Blood volume regulation during hemodialysis. Am. J. Kidney Dis. 32, 739-748 (1998).

10. Toussaint, N., Cooney, P. \& Kerr, P. G. Review of dialysate calcium concentration in hemodialysis. Hemodial. Int. 10, 326-337 (2006).

11. Magder, S. The meaning of blood pressure. Crit. Care 22, 257 (2018)

12. Riva, C. E., Grunwald, J. E. \& Petrig, B. L. Autoregulation of human retinal blood flow. An investigation with laser Doppler velocimetry. Investig. Ophthalmol. Vis. Sci. 27, 1706-1712 (1986).

13. Coppolino, G., Pisano, A., Rivoli, L. \& Bolignano, D. Renal denervation for resistant hypertension. Cochrane Database Syst. Rev. 2, 011499 (2017).

14. Lee, W. H. et al. Retinal microvascular change in hypertension as measured by optical coherence tomography angiography. Sci. Rep. 9, 156 (2019).

15. Carnevali, A. et al. Optical coherence tomography angiography analysis of retinal vascular plexuses and choriocapillaris in patients with type 1 diabetes without diabetic retinopathy. Acta Diabetol. 54, 695-702 (2017).

16. Flammer, J. et al. The eye and the heart. Eur. Heart J. 34, 1270-1278 (2013). 
17. Liew, G., Wong, T. Y., Mitchell, P., Cheung, N. \& Wang, J. J. Retinopathy predicts coronary heart disease mortality. Heart 95 , 391-394 (2009).

18. Kaiser, H. J. \& Flammer, J. Systemic hypotension: A risk factor for glaucomatous damage?. Ophthalmologica 203, 105-108 (1991).

19. Okumura, Y., Yuki, K. \& Tsubota, K. Low diastolic blood pressure is associated with the progression of normal-tension glaucoma. Ophthalmologica 228, 36-41 (2012).

20. Shin, Y. U. et al. Optical coherence tomography angiography analysis of changes in the retina and the choroid after haemodialysis. Sci. Rep. 8, 17184 (2018).

21. Zhang, Y., Weng, H., Li, Q. \& Wang, Z. Changes in retina and choroid after haemodialysis assessed using optical coherence tomography angiography. Clin. Exp. Optom. 101, 674-679 (2018).

22. Coppolino, G. et al. Experimental therapies in renal replacement: The effect of two different potassium acetate-free biofiltration protocols on striated muscle fibers. Ther. Apher Dial. 11, 375-381 (2007).

23. Assimon, M. M. \& Flythe, J. E. Definitions of intradialytic hypotension. Semin. Dial 30, 464-472 (2017).

24. Huang, D., Jia, Y., Gao, S. S., Lumbroso, B. \& Rispoli, M. Optical coherence tomography angiography using the optovue device. Dev. Ophthalmol. 56, 6-12 (2016).

25. Inrig, J. K. Beware intradialytic hypotension: How low is too low?. Clin. J. Am. Soc. Nephrol. 13, 1453-1454 (2018).

26. Bolignano, D. et al. Prognostic models in the clinical arena. Aging Clin. Exp. Res. 24, 300-304 (2012).

27. Tripepi, G. et al. An overview of standard statistical methods for assessing exposure-outcome link in survival analysis (part I): Basic concepts. Aging Clin. Exp. Res. 24, 109-112 (2012).

28. Abd ElHafeez, S. et al. An overview on standard statistical methods for assessing exposure-outcome link in survival analysis (Part II): The Kaplan-Meier analysis and the Cox regression method. Aging Clin. Exp. Res. 24, 203-206 (2012).

29. Battaglia Parodi, M. et al. Vessel density analysis in patients with retinitis pigmentosa by means of optical coherence tomography angiography. Br. J. Ophthalmol. 101, 428-432 (2017).

30. La Spina, C., Carnevali, A., Marchese, A., Querques, G. \& Bandello, F. Reproducibility and reliability of optical coherence tomography angiography for foveal avascular zone evaluation and measurement in different settings. Retina 37, 1636-1641 (2017).

31. De Vitis, L. A. et al. DualTrack technology improves optical coherence tomography angiography image quality. Ophthalm. Surg. Lasers Imaging Retina 48, 918-926 (2017).

32. Ulas, F. et al. Evaluation of choroidal and retinal thickness measurements using optical coherence tomography in non-diabetic haemodialysis patients. Int. Ophthalmol. 33, 533-539 (2013).

33. Theodossiadis, P. G. et al. Hemodialysis-induced alterations in macular thickness measured by optical coherence tomography in diabetic patients with end-stage renal disease. Ophthalmologica 227, 90-94 (2012).

34. Jung, J. W., Chin, H. S., Lee, D. H., Yoon, M. H. \& Kim, N. R. Changes in subfoveal choroidal thickness and choroidal extravascular density by spectral domain optical coherence tomography after haemodialysis: A pilot study. Br. J. Ophthalmol. 98, 207-212 (2014).

35. Chang, I. B., Lee, J. H. \& Kim, J. S. Changes in choroidal thickness in and outside the macula after hemodialysis in patients with end-stage renal disease. Retina 37, 896-905 (2017).

36. Kur, J., Newman, E. A. \& Chan-Ling, T. Cellular and physiological mechanisms underlying blood flow regulation in the retina and choroid in health and disease. Prog. Retin Eye Res. 31, 377-406 (2012).

37. Bruun, A., Ehinger, B., Sundler, F., Tornqvist, K. \& Uddman, R. Neuropeptide Y immunoreactive neurons in the guinea-pig uvea and retina. Investig. Ophthalmol. Vis. Sci. 25, 1113-1123 (1984).

38. Bill, A. \& Sperber, G. O. Control of retinal and choroidal blood flow. Eye (Lond.) 4(Pt 2), 319-325 (1990).

39. Underwood, C. F. et al. Uraemia: An unrecognized driver of central neurohumoral dysfunction in chronic kidney disease?. Acta Physiol. (Oxf.) 219, 305-323 (2017).

40. Pemp, B. \& Schmetterer, L. Ocular blood flow in diabetes and age-related macular degeneration. Can. J. Ophthalmol. 43, 295-301 (2008).

41. Tedeschi-Reiner, E., Strozzi, M., Skoric, B. \& Reiner, Z. Relation of atherosclerotic changes in retinal arteries to the extent of coronary artery disease. Am. J. Cardiol. 96, 1107-1109 (2005).

42. Liew, G. et al. Fractal analysis of retinal microvasculature and coronary heart disease mortality. Eur. Heart J. 32, 422-429 (2011).

43. Hughes, S. et al. Altered pericyte-endothelial relations in the rat retina during aging: implications for vessel stability. Neurobiol. Aging 27, 1838-1847 (2006).

44. Coppolino, G., Leonardi, G., Andreucci, M. \& Bolignano, D. Oxidative stress and kidney function: A brief update. Curr. Pharm. Des. 24, 4794-4799 (2018).

45. Leporini, C. et al. Effect of pentoxifylline on renal outcomes in chronic kidney disease patients: A systematic review and metaanalysis. Pharmacol. Res. 107, 315-332 (2016).

46. Bolignano, D., D’Arrigo, G., Pisano, A. \& Coppolino, G. Pentoxifylline for anemia in chronic kidney disease: A systematic review and meta-analysis. PLOS ONE 10, e0134104 (2015).

47. Gidday, J. M. \& Park, T. S. Adenosine-mediated autoregulation of retinal arteriolar tone in the piglet. Investig. Ophthalmol. Vis. Sci. 34, 2713-2719 (1993).

48. Holmgaard, K., Aalkjaer, C., Lambert, J. D., Hessellund, A. \& Bek, T. The relaxing effect of perivascular tissue on porcine retinal arterioles in vitro is mimicked by $\mathrm{N}$-methyl-D-aspartate and is blocked by prostaglandin synthesis inhibition. Acta Ophthalmol. 86, 26-33 (2008).

49. Tani, T., Nagaoka, T., Nakabayashi, S., Yoshioka, T. \& Yoshida, A. Autoregulation of retinal blood flow in response to decreased ocular perfusion pressure in cats: Comparison of the effects of increased intraocular pressure and systemic hypotension. Investig. Ophthalmol. Vis. Sci. 55, 360-367 (2014).

50. Osto, E., Coppolino, G., Volpe, M. \& Cosentino, F. Restoring the dysfunctional endothelium. Curr. Pharm. Des. 13, 1053-1068 (2007).

51. Paneni, F. et al. Deletion of the activated protein-1 transcription factor JunD induces oxidative stress and accelerates age-related endothelial dysfunction. Circulation 127(1229-1240), e1221 (2013).

52. Carracedo, J. et al. Mechanisms of cardiovascular disorders in patients with chronic kidney disease: A process related to accelerated senescence. Front. Cell Dev. Biol. 8, 185 (2020).

53. Yemisci, M. et al. Pericyte contraction induced by oxidative-nitrative stress impairs capillary reflow despite successful opening of an occluded cerebral artery. Nat. Med. 15, 1031-1037 (2009).

\section{Acknowledgements}

All Authors concurred with the submission and gave permission to be acknowledged. None of the Authors received financial or material support for the research and the work. Corresponding Authors had full access to all the data in the study and takes responsibility for the integrity of the data and the accuracy of the data analysis as well as the decision to submit for publication. 


\section{Author contributions}

G.C. and A.C.: Conceptualization; Data curation; Formal analysis; Investigation; Methodology; Project administration; Resources; Supervision; Writing_original draft; Writing_review \& editing. V.G., C.B., G.R., I.F. and G.P.: Data curation; Formal analysis; Investigation. M.A., G.F. and G.G.: Writing-review \& editing. V.S. and D.B.: Conceptualization; Data curation; Formal analysis; Investigation; Methodology; Project administration; Resources; Supervision; Writing_original draft; Writing—review \& editing.

\section{Competing interests}

The authors declare no competing interests.

\section{Additional information}

Correspondence and requests for materials should be addressed to A.C.

Reprints and permissions information is available at www.nature.com/reprints.

Publisher's note Springer Nature remains neutral with regard to jurisdictional claims in published maps and institutional affiliations.

(c) (i) Open Access This article is licensed under a Creative Commons Attribution 4.0 International

License, which permits use, sharing, adaptation, distribution and reproduction in any medium or format, as long as you give appropriate credit to the original author(s) and the source, provide a link to the Creative Commons licence, and indicate if changes were made. The images or other third party material in this article are included in the article's Creative Commons licence, unless indicated otherwise in a credit line to the material. If material is not included in the article's Creative Commons licence and your intended use is not permitted by statutory regulation or exceeds the permitted use, you will need to obtain permission directly from the copyright holder. To view a copy of this licence, visit http://creativecommons.org/licenses/by/4.0/.

(C) The Author(s) 2021 